\title{
Inhibition of mitochondrial respiration by phosphoenolpyruvate
}

\author{
C. Nathan Baily ${ }^{\mathrm{a}}$, Roger W. Cason ${ }^{\mathrm{a}}$, Shraddha S. Vadvalkar ${ }^{\mathrm{a}}$, Satoshi Matsuzaki ${ }^{\mathrm{a}}$, \\ Kenneth M. Humphries ${ }^{\mathrm{a}, \mathrm{b}, \mathrm{c}, *}$
}

${ }^{a}$ Free Radical Biology and Aging Research Program, Oklahoma Medical Research Foundation, Oklahoma City, OK, USA

${ }^{\mathrm{b}}$ Department of Molecular Biology and Biochemistry, University of Oklahoma Health Science Center, Oklahoma City, OK, USA

${ }^{\mathrm{c}}$ Reynolds Oklahoma Center on Aging, University of Oklahoma Health Science Center, Oklahoma City, OK, USA

\section{A R T I C L E I N F O}

\section{Article history:}

Received 17 June 2011

and in revised form 2 August 2011

Available online 16 August 2011

\section{Keywords:}

Cardiac mitochondria

Phosphoenolpyruvate

Oxidative phosphorylation

Free radicals

\begin{abstract}
A B S T R A C T
The cytosolic factors that influence mitochondrial oxidative phosphorylation rates are relatively unknown. In this report, we examine the effects of phosphoenolpyruvate (PEP), a glycolytic intermediate, on mitochondrial function. It is reported here that in rat heart mitochondria, PEP delays the onset of state 3 respiration in mitochondria supplied with either NADH-linked substrates or succinate. However, the maximal rate of state 3 respiration is only inhibited when oxidative phosphorylation is supported by NADH-linked substrates. The capacity of PEP to delay and/or inhibit state 3 respiration is dependent upon the presence or absence of ATP. Inhibition of state 3 is exacerbated in uncoupled mitochondria, with a $40 \%$ decrease in respiration seen with $0.1 \mathrm{mM}$ PEP. In contrast, ATP added exogenously or produced by oxidative phosphorylation completely prevents PEP-mediated inhibition. Mechanistically, the results support the conclusion that the main effects of PEP are to impede ADP uptake and inhibit NADH oxidation. By altering the $\mathrm{NADH} / \mathrm{NAD}^{+}$status of mitochondria, it is demonstrated that PEP enhances succinate dehydrogenase activity and increase free radical production. The results of this study indicate PEP may be an important modulator of mitochondrial function under conditions of decreased ATP.
\end{abstract}

(c) 2011 Elsevier Inc. All rights reserved.

\section{Introduction}

Glycolysis generates pyruvate, an oxidizable substrate that can be further metabolized in the mitochondria by pyruvate dehydrogenase to generate acetyl-CoA. Acetyl-CoA enters the Krebs cycle and generates reducing equivalents, in the form of NADH and succinate, for fueling the electron transport chain. When Krebs cycle intermediates accumulate, indicating a surplus of energy reserves or a reduced means of consuming oxygen, the rate of glycolysis is attenuated via feedback mechanisms. For example, citrate derived from the Krebs cycle inhibits cytosolic phosphofructose kinase 1 and 2 [1], critical regulatory steps of the overall rate of glucose oxidation. Thus, the ability of Krebs cycle activity to modulate glycolysis is well documented. However, it is relatively unknown how glycolytic intermediates may reciprocally regulate or affect mitochondrial functions and processes.

Amongst the glycolytic intermediates, phosphoenolpyruvate $(\mathrm{PEP})^{1}$ is of particular interest, in part, because of its permeability

\footnotetext{
* Corresponding author at: Free Radical Biology and Aging Research Program, Oklahoma Medical Research Foundation, 825 N.E. 13th Street, Oklahoma City, OK 73104, USA. Fax: +1 4052711437.

E-mail address: Kenneth-Humphries@omrf.org (K.M. Humphries).

${ }^{1}$ Abbreviations used: PEP, phosphoenolpyruvate; RCR, respiratory control ratio; SDH, succinate dehydrogenase; PEPCK-M, phosphoenolpyruvate; TMRM, tetramethylrhodamine; TTFA, thenoyltrifluoroacetone; ANT, adenine nucleotide transporter; $\mathrm{MDH}$, malate dehydrogenase.
}

through the mitochondrial inner membrane [2-5]. In the liver PEP is primarily transported by a tricarboxylate carrier, while in the heart it is primarily transported by the adenine nucleotide transporter [3]. In gluconeogenic tissues mitochondria are an important source of PEP, where it is produced via phosphoenolpyruvate carboxykinase (PEPCK-M) and then transported into the cytosol [6]. Importantly, PEP, but not other glycolytic intermediates, has also been described to have effects on various mitochondrial processes. For example, treatment of isolated rat heart mitochondria with PEP causes an efflux of calcium $[3,7,8]$ and can induce opening of the mitochondrial permeability transition pore [9]. PEP has also been reported to inhibit mitochondrial protein synthesis in liver $[10,11]$ and $\alpha$-glycerophosphate dehydrogenase activity in rat skeletal muscle [12]. Nevertheless, relatively little is known about the effects that PEP may have on oxidative phosphorylation and bioenergetic processes.

In the present work, detailed analyses of the effects of PEP on isolated rat heart mitochondria were performed. It is demonstrated that PEP inhibits NADH-linked respiration, but not succinatelinked respiration. This inhibition was greatly enhanced in uncoupled mitochondria and attenuated by ATP. Mechanistically, the reduced rate of oxidative phosphorylation is accompanied by a decreased rate of $\mathrm{NAD}(\mathrm{P}) \mathrm{H}$ consumption upon transitioning to state 3 respiration and is consistent with an inhibition of complex I. The decreased rate of electron transport chain activity, in turn, leads to an increase in free radical production. 


\section{Materials and methods}

\section{Isolation of cardiac mitochondria}

Sprague Dawley rats were euthanized by decapitation. Hearts were excised immediately and placed into $20 \mathrm{~mL}$ of ice-cold buffer containing $210 \mathrm{mM}$ mannitol, $70 \mathrm{mM}$ sucrose, $1.0 \mathrm{mM}$ EDTA, $5.0 \mathrm{mM}$ MOPS, pH 7.4 (buffer A). Hearts were perfused with $10 \mathrm{~mL}$ of buffer $\mathrm{A}$ and then minced with scissors. This was followed by Polytron homogenization. The homogenate was then spun at $500 \mathrm{~g}$ for $5 \mathrm{~min}$ at $4{ }^{\circ} \mathrm{C}$, the supernatant collected and passed through cheesecloth, and spun again for $10 \mathrm{~min}$ at $5000 \mathrm{~g}$. The resulting mitochondrial pellet was resuspended in approximately $200 \mu \mathrm{L}$ of buffer $\mathrm{A}$ and protein concentration determined by the BCA method (Pierce) using BSA as a standard.

\section{Mitochondrial respiration}

Mitochondria were diluted to $0.25 \mathrm{mg} / \mathrm{mL}$ in $210 \mathrm{mM}$ mannitol, $70 \mathrm{mM}$ sucrose, $5.0 \mathrm{mM} \mathrm{KH}_{2} \mathrm{PO}_{4}$, and $10 \mathrm{mM}$ MOPS, pH 7.4 (buffer B) containing either $10 \mathrm{mM}$ glutamate $/ 2.5 \mathrm{mM}$ malate, or $30 \mu \mathrm{M}$ palmitoyl carnitine $/ 0.1 \mathrm{mM}$ malate, or $10 \mathrm{mM}$ succinate. Respiration was measured polarographically at $25^{\circ} \mathrm{C}$ with a Clark-style oxygen electrode (Instech) in the presence or absence of PEP (Sigma Aldrich) and indicated reagents. State 3 respiration [13] was initiated by addition of ADP $(0.5 \mathrm{mM})$ or $30 \mu \mathrm{M}$ DNP at $2.0 \mathrm{~min}$. The starting amount of molecular oxygen in the $0.6 \mathrm{~mL}$ electrode chamber was based on the assumption that $265 \mathrm{nmol}$ of molecular oxygen are dissolved per milliliter at atmospheric pressure and $20^{\circ} \mathrm{C}$.

\section{$N A D(P) H$ and membrane potential measurements}

Mitochondria were diluted to $0.25 \mathrm{mg} / \mathrm{mL}$ in buffer B with indicated substrates and PEP concentrations. NAD $(\mathrm{P}) \mathrm{H}$ autofluorescence was measured using $340 \mathrm{~nm}$ excitation $/ 460 \mathrm{~nm}$ emission [14]. NADH and NADPH autofluorescence are indistinguishable, and therefore measurements are referred to as $\mathrm{NAD}(\mathrm{P}) \mathrm{H}$. Membrane potential was measured using tetramethylrhodamine methyl ester (TMRM, Molecular Probes), a cationic dye that is accumulated in mitochondria as a function of the membrane potential [15]. Increased uptake of the dye is accompanied by a decreased fluorescence, while depolarization causes a net efflux and increase in fluorescence. Changes in TMRM fluorescence were monitored using $549 \mathrm{~nm}$ excitation/575 nm emission.

\section{Succinate dehydrogenase activity}

Mitochondria were diluted to $0.25 \mathrm{mg} / \mathrm{mL}$ in buffer B with $10 \mathrm{mM}$ succinate and indicated amounts of PEP and ADP. At times indicated, samples were diluted 1:10 in a buffer containing $25 \mathrm{mM}$ MOPS pH 7.2 and $0.025 \%$ Triton X-100. It was found that this detergent solubilization methodology effectively locked complex II into either the active or inactive state that was present in the intact mitochondria [16]. Complex II activity was measured spectrophotometrically by the thenoyltrifluoroacetone (TTFA) sensitive reduction of ubiquinone-1 $(50 \mu \mathrm{M})$ at $280 \mathrm{~nm}\left(\varepsilon=13,700 \mathrm{M}^{-1} \mathrm{~cm}^{-1}\right)$ [16]. The presence of $0.025 \%$ Triton X-100 completely blocked ubiquinone reduction by complex I [17] and ubiquinol oxidation by complex III.

\section{$\mathrm{H}_{2} \mathrm{O}_{2}$ production}

Mitochondria were diluted to $0.125 \mathrm{mg} / \mathrm{mL}$ in buffer B with $10 \mathrm{mM}$ glutamate and $2.5 \mathrm{mM}$ malate, or $10 \mathrm{mM}$ succinate, and indicated concentrations of PEP and ADP. Amplex Red (Invitrogen) was added at a concentration of $20 \mu \mathrm{M}$ and the increase in fluorescence measured over time ( $574 \mathrm{~nm}$ excitation/584 nm emission). After achieving a baseline rate, the increase in fluorescence was measured upon addition of $4.0 \mathrm{U} / \mathrm{mL}$ horseradish peroxidase.

\section{Statistical analysis}

Data are presented as means \pm SD. The data were evaluated utilizing a two-tailed $t$ test. Statistical significance was assigned for $p \leqslant 0.05$, as indicated.

\section{Results and discussion}

\section{The effect of PEP on mitochondrial respiration}

Initial experiments were undertaken to examine the effects of PEP on mitochondrial respiration. As shown in Fig. 1A, rat heart mitochondria utilizing glutamate/malate as substrates and incubated with $1.0 \mathrm{mM}$ PEP display two specific alterations in oxidative phosphorylation. Firstly, as compared to untreated mitochondria, there is a slow transition into state 3 respiration upon addition of ADP. Secondly, a $20 \%$ decrease in the rate of state 3 respiration was observed (Figs. 1A and 1C). This inhibition of state 3 was not accompanied by decreases in state 4 or the ADP/O ratio. PEP inhibited palmitoylcarnitine-linked respiration in a similar manner and to a similar magnitude ( $30 \%$ decrease, Fig. 1C). In contrast to NADH-supported substrates, PEP had a different effect on succinate-linked respiration. As shown in Fig. 1A, PEP also delayed the onset of state 3 respiration upon addition of ADP, but had no effect on the maximal rate of oxygen consumption (Fig. 1C).

The PEP-induced slow transition to state 3 respiration observed with all tested oxidizable substrates could be due, in part, to impaired uptake of ADP by the adenine nucleotide transporter (ANT). The effect of PEP on uncoupled respiration, which occurs independently of ANT activity, was therefore examined. As shown in Figs. 1B and 1C, the effect of PEP on glutamate/malate supported respiration was exacerbated in uncoupled mitochondria, with $1.0 \mathrm{mM}$ PEP inhibiting state 3 respiration by $50 \%$ percent. Similarly, palmitoylcarnitine supported uncoupled respiration was inhibited by $60 \%$ (Fig. 1C). In contrast, maximal succinate-supported uncoupled respiration was unaffected by this concentration of PEP (Figs. $1 \mathrm{~B}$ and $1 \mathrm{C}$ ). Importantly, it was observed that neither succinate nor $\mathrm{NADH}$-supported respiration exhibited delayed onset of maximal respiratory rates upon addition of uncoupler (Fig. 1B). This suggests PEP has multiple effects upon oxidative phosphorylation. Firstly, it impedes coupled respiration through the inhibition ADP uptake. Secondly, the differential sensitivity of NADH versus succinate supported respiration indicates either inhibition of NADH production by mitochondrial dehydrogenases, or consumption of NADH by complex I.

To explore the potency of PEP in inhibiting mitochondrial respiration, experiments were next performed to determine the concentration dependence. As shown in Fig. 2, maximal inhibition of coupled respiration (using glutamate/malate as substrates) was reached at $100 \mu \mathrm{M}$ PEP, with inhibition not increasing above $30 \%$ with higher concentrations. In contrast, uncoupled mitochondria exhibited a more drastic and concentration dependent inhibition. $250 \mu \mathrm{M}$ PEP inhibited uncoupled state 3 respiration nearly $50 \%$, with minimal additional effects seen above this concentration.

\section{ATP prevents PEP-induced inhibition of state 3 respiration}

Experiments were next undertaken to discern the differential sensitivity of coupled and uncoupled respiration to PEP-induced 

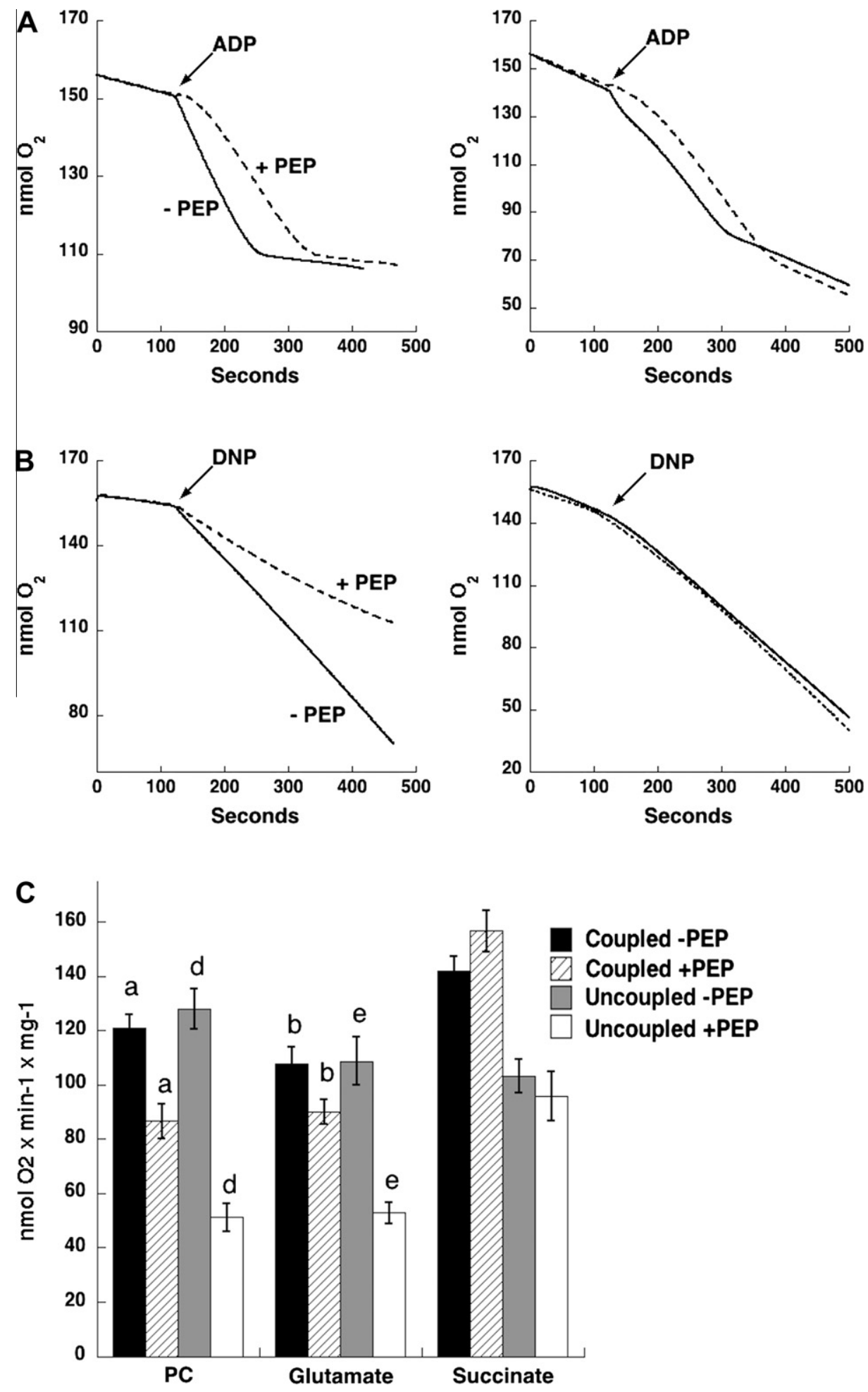

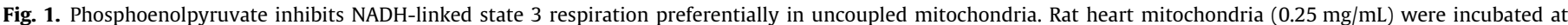

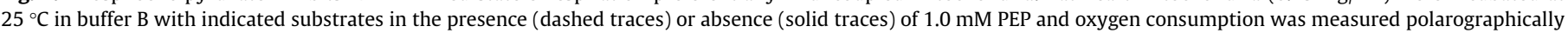

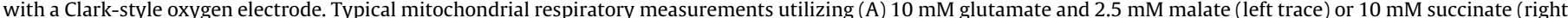

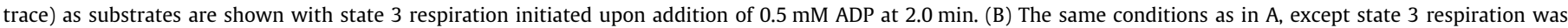

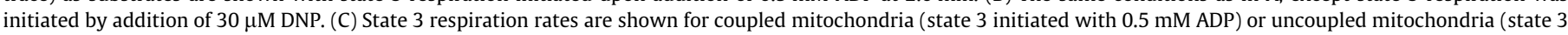

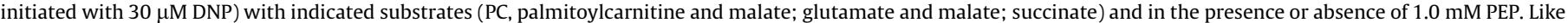
symbols indicate statistically significant differences between the two samples by student's $t$ test $(p<0.05, n \geqslant 5$ for each experimental condition).

inhibition. We hypothesized that ATP, produced in coupled mitochondria during state 3 respiration, may be an important determinant in attenuating PEP-mediated inhibition. Experiments were therefore performed in which ATP and PEP were added to mitochondria utilizing glutamate/malate prior to initiation of state 3. As shown in Fig. 3, the inclusion of ATP completely prevented PEP-induced state 3 inhibition in coupled (Fig. 3A) and uncoupled (Fig. 3B) mitochondria. Importantly, this result indicates that 


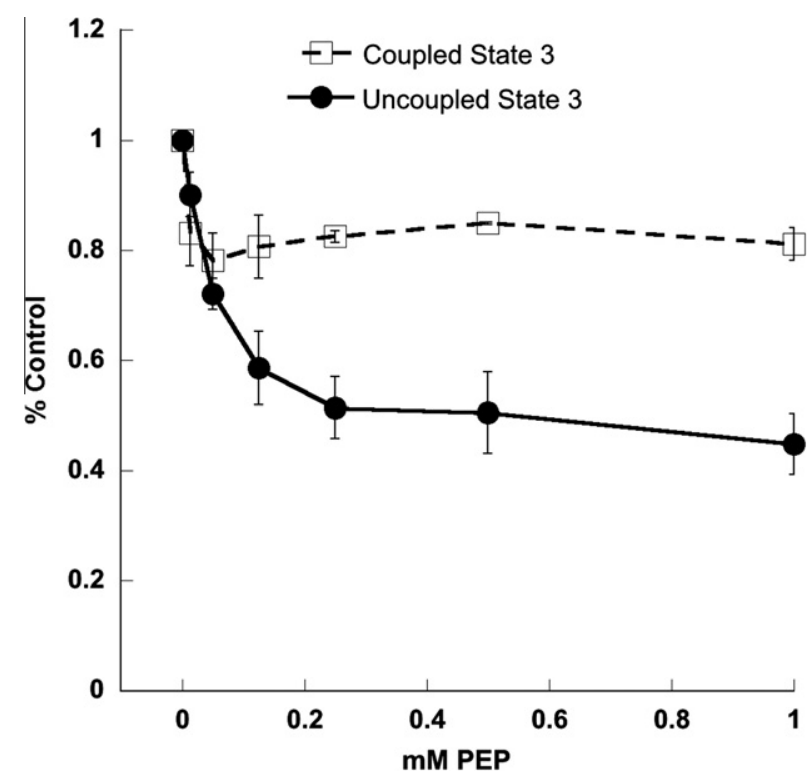

Fig. 2. Uncoupled state 3 respiration is more sensitive to PEP-mediated inhibition. Mitochondria, utilizing $10 \mathrm{mM}$ glutamate and $2.5 \mathrm{mM}$ malate as substrates, were incubated with indicated concentrations of PEP and state 3 respiration initiated by addition of either $0.5 \mathrm{mM}$ ADP (open squares) or $30 \mu \mathrm{M}$ DNP (closed circles) as indicated in Fig. 1 ( $n \geqslant 5$ for each experimental condition).

mitochondria would be particularly susceptible to inhibition under conditions of reduced ATP.

The mechanism whereby ATP prevents PEP-induced inhibition appears enzymatic in nature. This was demonstrated by experiments performed in the presence of AMP-PNP, a non-hydrolyzable analog of ATP. In contrast to the protection by ATP (Fig. 3A), it was found that AMP-PNP did not prevent PEP-mediated inhibition (data not shown). It has been previously shown that the ANT in heart mitochondria are the primary transporters of PEP, and that this transport is blocked by ATP [3]. Thus, we hypothesized that ATP may be serving to inhibit PEP uptake under our experimental conditions. We therefore measured the PEP concentration in the external mitochondrial milieu at the start and end of incubation conditions by a pyruvate kinase and lactate dehydrogenase coupled reaction. It was found that, regardless of the presence of ATP, there was a surprisingly little decrease in PEP concentrations (data not shown). Thus, the protection afforded by ATP does not appear to be due to an accelerated rate of PEP removal or metabolism. We concluded that perhaps a relatively small uptake of PEP, below our detection limits, is sufficient to induce the observed changes in mitochondrial respiration. Alternately, PEP may also have some effect on mitochondrial function without being transported into the matrix. For example, it is possible that PEP inhibits substrate uptake in an ATP-sensitive manner.

\section{PEP reduces the rate of mitochondrial consumption of $N A D(P) H$}

To gain further insight into the mechanism whereby PEP inhibits mitochondrial respiration, the production and consumption of $\mathrm{NAD}(\mathrm{P}) \mathrm{H}$ was examined via fluorescence spectroscopy. As shown in Fig. 4A, under control conditions the addition of ADP to mitochondria supplied with glutamate/malate stimulates state 3 respiration, and this coincides with an extremely rapid oxidation of $\mathrm{NAD}(\mathrm{P}) \mathrm{H}$. In contrast, we noted multiple effects on $\mathrm{NAD}(\mathrm{P}) \mathrm{H}$ oxidation in the presence of $1.0 \mathrm{mM}$ PEP. Firstly, the steady state levels of $\mathrm{NAD}(\mathrm{P}) \mathrm{H}$ prior to ADP addition and during state 3 respiration were elevated in PEP treated mitochondria as compared to controls, suggesting either increased rates of $\mathrm{NAD}^{+}$reduction or decreased rates NADH oxidation. Secondly, there was a decreased rate of $\mathrm{NAD}(\mathrm{P}) \mathrm{H}$ oxidation upon addition of ADP, consistent with the slow transition into state 3 respiration as measured by oxygen consumption. Finally, corresponding to the slower rate of state 3 respiration displayed in Fig. 1A, there was a delayed return to steady state levels of $\mathrm{NAD}(\mathrm{P}) \mathrm{H}$ during state 4 respiration as compared to untreated controls. These three changes are consistent with both inhibition of ADP uptake and impaired NADH oxidation. The protective effect of ATP in preventing PEP-mediated inhibition were also reflected in the $\mathrm{NAD}(\mathrm{P}) \mathrm{H}$ profile. As shown in Fig. 4B, the profile of $\mathrm{NAD}(\mathrm{P}) \mathrm{H}$ consumption induced by ADP (state 3 ) and recovery upon transition to state 4 were identical in PEP treated and control mitochondria when in the presence of ATP.

Alterations in mitochondrial respiration and $\mathrm{NAD}(\mathrm{P}) \mathrm{H}$ utilization may be accompanied by perturbations in the mitochondrial membrane potential. The effect of PEP on membrane potential was therefore examined by monitoring TMRM fluorescence [15]. As shown in Fig. 4C, control mitochondria show a rapid increase in TMRM fluorescence upon addition of ADP, indicative of the transition into state 3 respiration and mitochondrial depolarization. Upon returning to state 4, there is a decrease in fluorescence as mitochondria repolarize. In the presence of $1.0 \mathrm{mM}$ PEP, the rate of depolarization upon addition of ADP is decreased as compared to controls, while the rate of repolarization is unchanged. Importantly, the fact that PEP treatment has no effects on membrane
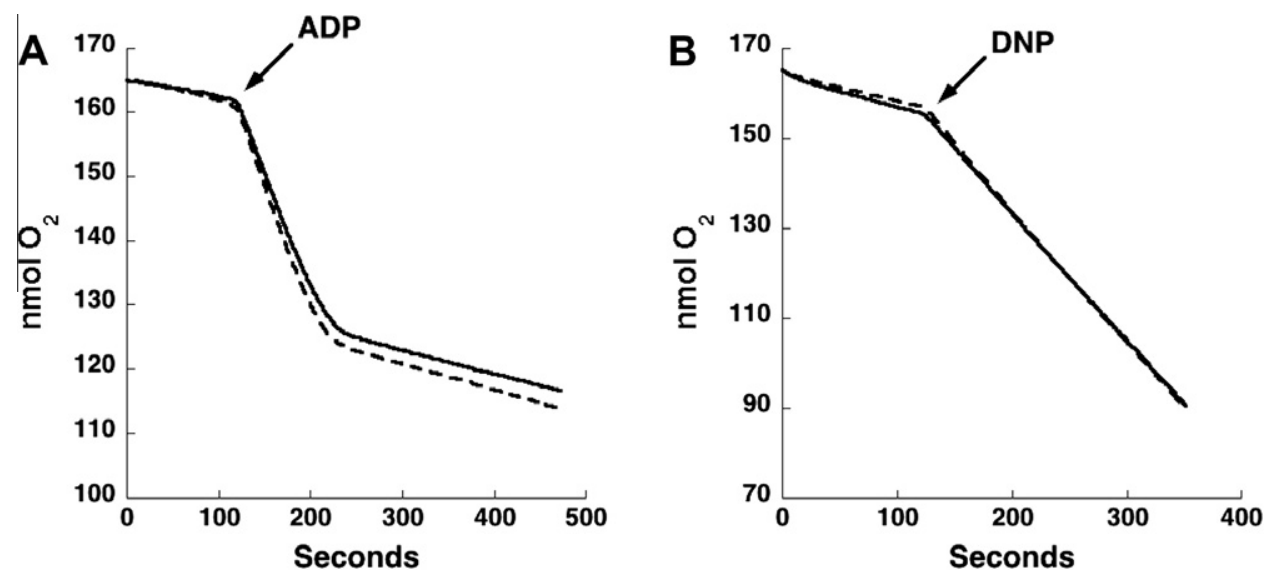

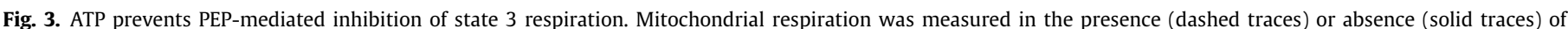

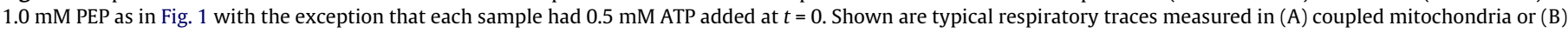
uncoupled mitochondria ( $0.5 \mathrm{mM}$ ADP or $30 \mu \mathrm{M}$ DNP added at $2.0 \mathrm{~min}$, respectively). 

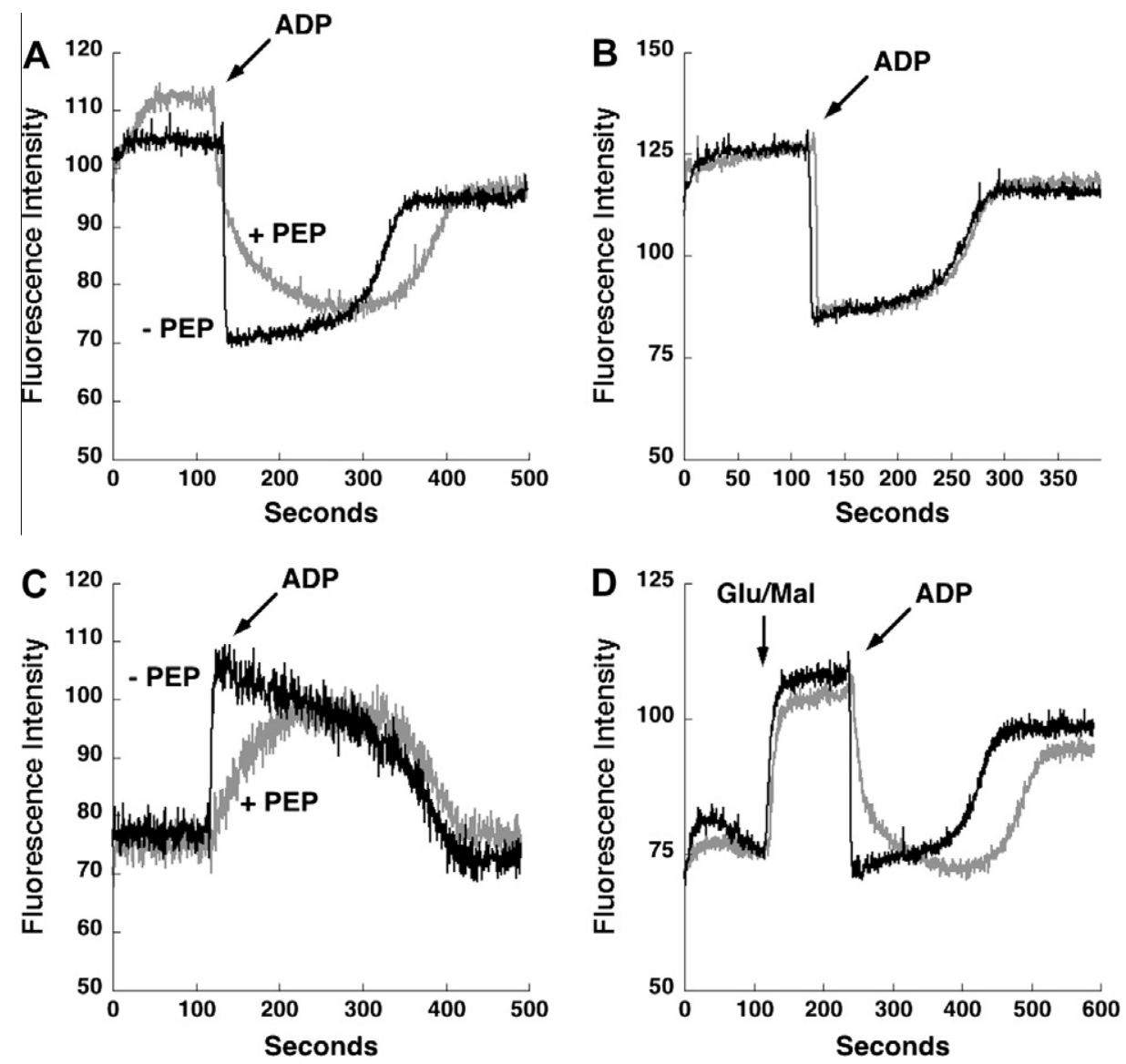

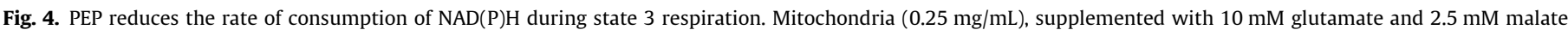

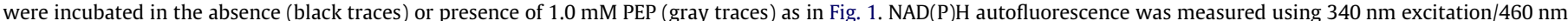

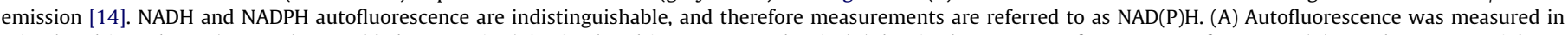

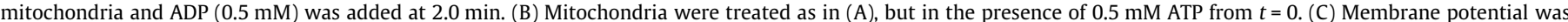

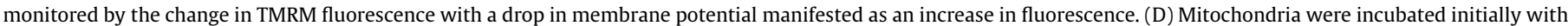
or without $1.0 \mathrm{mM}$ PEP and respiratory substrates (glutamate/malate) added at $2.0 \mathrm{~min}$. ADP $(0.5 \mathrm{mM})$ was then added at $4.0 \mathrm{~min}$.

potential during state 2 , only on conditions of maximum respiration, indicates mitochondrial integrity is intact under the experimental conditions.

Our findings clearly demonstrate PEP alters NAD $(\mathrm{P}) \mathrm{H}$ consumption during state 3 respiration. As mentioned above, inhibition of $\mathrm{NAD}(\mathrm{P}) \mathrm{H}$ oxidation observed during state 3 respiration could either be a factor of decreased NADH oxidation by the electron transport chain, or a decreased reduction of $\mathrm{NAD}^{+}$by mitochondrial dehydrogenases. To determine which process is affected, mitochondria were pre-incubated with or without PEP prior to addition of glutamate and malate. As shown in Fig. 4D addition of glutamate/malate to mitochondria induces the same rate of $\mathrm{NAD}(\mathrm{P}) \mathrm{H}$ production in both treated and control mitochondria. Interestingly, both samples had similar levels of $\mathrm{NAD}(\mathrm{P}) \mathrm{H}$ prior to the addition of ADP in these experiment, indicating the effect of PEP was somewhat dependent upon the order of addition. Nevertheless, the rate of $\mathrm{NAD}(\mathrm{P}) \mathrm{H}$ consumption was inhibited in PEP treated mitochondria upon addition of ADP. This results supports the conclusion that the effect induced by PEP is specific for $\mathrm{NAD}(\mathrm{P}) \mathrm{H}$ consumption.

\section{The effect of PEP on succinate-linked respiration}

The results presented in Fig. 1A demonstrate PEP delays the onset of state 3 respiration when succinate is the substrate, but has no effect on the maximal rate of respiration. In the presence of succinate, mitochondria produce a large amount of NADH that is attributable to reverse electron flow [18]. It was therefore expected that the alteration in $\mathrm{NAD}(\mathrm{P}) \mathrm{H}$ oxidation seen with $\mathrm{NADH}-$ linked substrates would also reflected by changes when succinate as a substrate. As shown in Fig. 5A, in the presence of PEP, similar to $\mathrm{NADH}$-linked substrates there is a marked decrease in the rate of $\mathrm{NAD}(\mathrm{P}) \mathrm{H}$ consumption upon addition of ADP. Furthermore, there is a sustained elevation in $\mathrm{NAD}(\mathrm{P}) \mathrm{H}$ levels during state 3 as compared to control mitochondria. Thus, while there is no overt change in the rate of respiration, PEP nevertheless disrupts the normal status of $\mathrm{NAD}(\mathrm{P}) \mathrm{H}$ oxidation and reduction when succinate is the substrate.

A change in the redox status induced by PEP is likely to alter those enzymatic activities, such as succinate dehydrogenase (SDH, or complex II), that are sensitive to the [NADH]/[NAD $\left.{ }^{+}\right]$ratio. We and others have previously shown that sustaining the [NADH]/ $\left[\mathrm{NAD}^{+}\right]$ratio during state 3 respiration, such as with the complex I inhibitor rotenone, enhances SDH activity [16]. This is attributable to the prevention of the malate dehydrogenase (MDH) driven production of oxaloacetate, a potent inhibitor of SDH $[16,19]$, which only occurs when the ratio of $[\mathrm{NADH}] /\left[\mathrm{NAD}^{+}\right]$is significantly reduced. Experiments were therefore undertaken to determine the effect of PEP on SDH activity. As shown in Fig. 5B, under control conditions the activity of SDH decreases by 65\% during state 3 respiration as compared to activity prior to addition of ADP. This dramatic decrease in SDH activity during state 3 is consistent with previous reports of SDH being regulated by the metabolic status of mitochondria. However, in the presence of PEP, SDH activity only decreases by $12 \%$ during state 3 respiration. No change in 

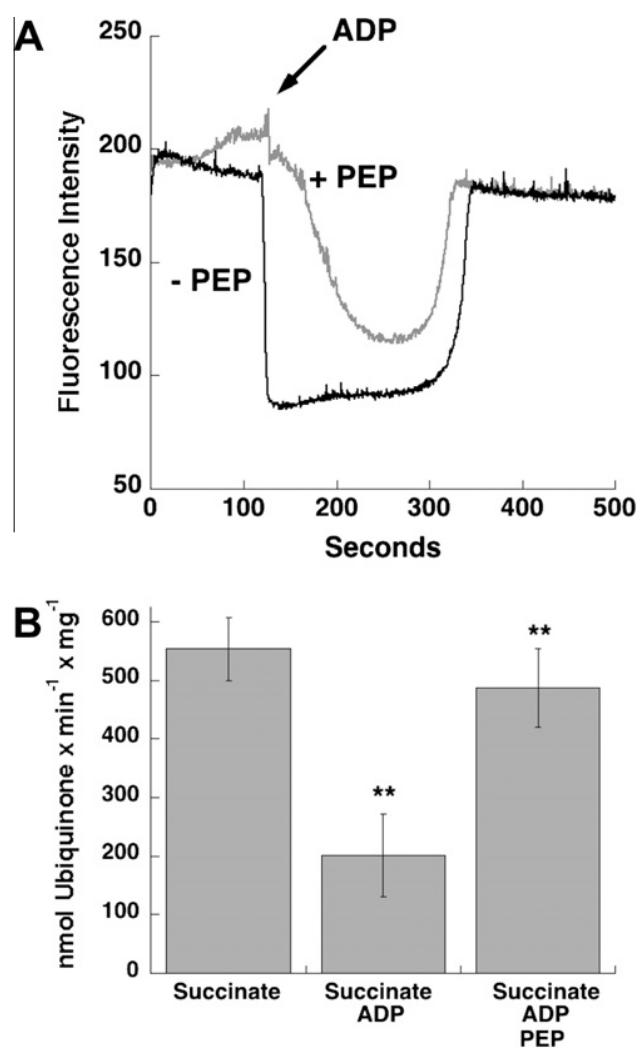

Fig. 5. PEP reduces the rate of $N A D(P) H$ oxidation and sustains complex II in the active state during state 3 respiration. (A) Mitochondrial autofluorescence was measured as in Fig. 4, with mitochondria utilizing succinate $(10 \mathrm{mM})$ as substrate and in the presence (gray trace) or absence (black trace) of $1.0 \mathrm{mM} \mathrm{PEP.} \mathrm{(B)}$ Mitochondria $(0.25 \mathrm{mg} / \mathrm{mL})$ in buffer B with $10 \mathrm{mM}$ succinate were incubated with or without $1.0 \mathrm{mM}$ PEP and ADP $(0.5 \mathrm{mM})$ was added at $2.0 \mathrm{~min}$ to initiate state 3 respiration as indicated. At $3.0 \mathrm{~min}, 100 \mu \mathrm{L}$ of sample was removed and diluted 1:10 into a buffer containing $0.025 \%$ Triton X-100 and complex II activity measured spectrophotometrically by monitoring ubuqinone- 1 reduction. Like symbols indicate statistically significant differences between the two samples by student's $t$ test $(p<0.005, n=5)$

SDH activity was seen in PEP treated versus control mitochondria prior to the addition of ADP (not shown). Thus, an important effect of PEP-mediated inhibition of NADH oxidation is to (1) prevent accumulation of oxaloacetate; and (2) maintain SDH in an active state even in the presence of ADP.

\section{PEP increases hydrogen peroxide production}

The rate at which mitochondria produce free radicals may be modulated by changes in respiratory activity. Specifically, it is known that inhibition of the electron transport chain activities increases superoxide production via an increase in the half-life of the reduced state of respiratory chain electron carriers. Because PEP reduces the rate of $\mathrm{NAD}(\mathrm{P}) \mathrm{H}$ oxidation, it was hypothesized that it would also increases the rate of superoxide production. The effect of PEP on mitochondrial superoxide production, as measured by $\mathrm{H}_{2} \mathrm{O}_{2}$ release using Amplex Red, was therefore examined. Consistent with previous reports, the rate $\mathrm{H}_{2} \mathrm{O}_{2}$ production is very low in mitochondria utilizing glutamate and malate as substrates (43.4 $\mathrm{pmol} \mathrm{H}_{2} \mathrm{O}_{2} \times \mathrm{min}^{-1} \times \mathrm{mg}^{-1}$ ), and was unaffected by the presence of $1.0 \mathrm{mM}$ PEP $\left(42.7 \mathrm{pmol} \mathrm{H}_{2} \mathrm{O}_{2} \times \mathrm{min}^{-1} \times \mathrm{mg}^{-1}\right)$. This result was somewhat surprising given that PEP inhibits NADH-linked respiration. Nevertheless, this rate of $\mathrm{H}_{2} \mathrm{O}_{2}$ production was much greater in mitochondria utilizing succinate $(270.6 \pm 94.0 \mathrm{pmol}$ $\mathrm{H}_{2} \mathrm{O}_{2} \times \min ^{-1} \times \mathrm{mg}^{-1}$ ), and exacerbated by the presence of 1.0 mM PEP (374.9 \pm 147.5 pmol $\mathrm{H}_{2} \mathrm{O}_{2} \times \mathrm{min}^{-1} \times \mathrm{mg}^{-1}$ ) (Fig. 6).
While there was a large day-to-day variation in the rate of $\mathrm{H}_{2} \mathrm{O}_{2}$ production in mitochondria, the increase in production induced by PEP was consistently observed (37.8\% increase $\pm 9.3 \%, n=4$ ). During state 3 respiration, the rate of $\mathrm{H}_{2} \mathrm{O}_{2}$ production was significantly reduced regardless of the presence of PEP (Fig. 6). The discrepancy between the effect of PEP on $\mathrm{H}_{2} \mathrm{O}_{2}$ production with either $\mathrm{NADH}$ or succinate-linked substrates may be attributable to the distinct mechanisms by which these substrates generate superoxide [18]. NADH-linked substrates generate superoxide within complex I at distinct redox centers by the forward flow of electrons from NADH to FMN, to FeS centers, to ubiquinone. In contrast, succinate generates superoxide by reverse electron flow through complex I to reduce NADH. Reverse electron flow occurs when the ubiquinone pool is highly reduced and the membrane potential is intact [20]. We speculate that PEP enhances the reverse electron flow mediated superoxide production uniquely, perhaps through an interaction between PEP and the highly reduced ubiquinone pool produced in the presence of succinate.

\section{Mechanism and significance}

Our results suggest PEP is inhibiting state 3 respiration via multiple mechanisms. Firstly, the delayed onset of coupled, but not uncoupled, state 3 respiration seen with both NADH and succinate-linked substrates is indicative of inhibition of ADP uptake. A second major mechanism is likely through inhibition of the electron transport chain at complex I. This mechanism is supported strongly by the observation that the maximal rates of state 3 respiration are decreased in mitochondria utilizing glutmate/malate or palmitoylcarnitine as respiratory substrates, but not succinate. It is also supported by the finding that PEP maintains high SDH activity during state 3 respiration, indicative of decreased complex I activity. Nevertheless, assaying complex I activity or NADH oxidase activity (an overall assessment of electron transport chain function) displayed no differences in activity in mitochondria treated with PEP (data not shown). To perform these assays, mitochondria were disrupted either by freezing and thawing or sonication and activities were measured by standard UV/Vis spectroscopy techniques [21]. This suggests the decreased rate of complex I observed in the presence of PEP requires intact mitochondria that are able to sustain a membrane potential.

The heart relies almost exclusively on $\beta$-oxidation for the generation of ATP, but nevertheless has the capacity to switch to

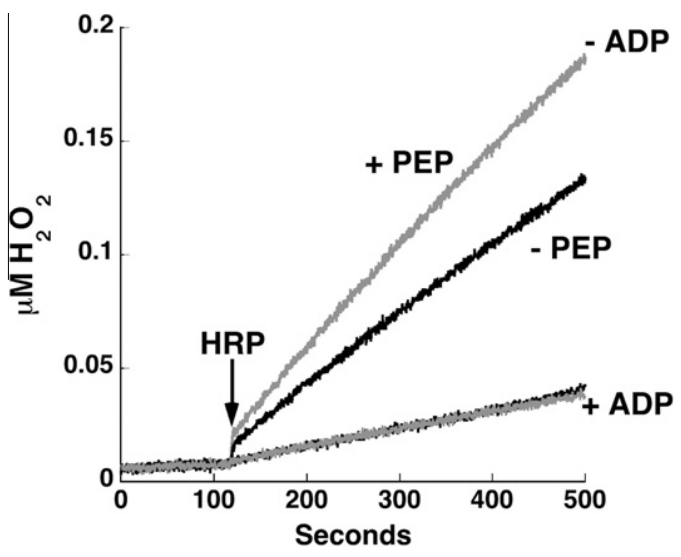

Fig. 6. PEP increases the rate of superoxide production in mitochondria. The rate of superoxide production was estimated by fluorometrically $(574 \mathrm{~nm}$ excitation/ $584 \mathrm{~nm}$ emission) measuring the efflux of $\mathrm{H}_{2} \mathrm{O}_{2}$ from mitochondria. Mitochondrial samples $(0.25 \mathrm{mg} / \mathrm{mL})$ were incubated in buffer B with $10 \mathrm{mM}$ succinate, $20 \mu \mathrm{M}$ Amplex Red, and ADP ( $0.5 \mathrm{mM}$ added at $t=0$, as indicated) in the presence (gray traces) or absence (black traces) of $1.0 \mathrm{mM}$ PEP. After a baseline reading was determined, the rate of $\mathrm{H}_{2} \mathrm{O}_{2}$ production was assessed upon addition of $4.0 \mathrm{U} \mathrm{HRP}$ at $2.0 \mathrm{~min}$. Typical measurements are shown $(n=4)$. 
glycolysis as the primary energy source, especially under disease and stress conditions such as ischemia [1,22-24]. Indeed, glucose uptake increases with ischemia, and efficient utilization of glycolysis is an important determinant in reducing ischemia and reperfusion injury [23-25]. However, the actual effects of ischemia on glycolysis and glycolytic intermediate concentrations are not clear, and are likely dependent upon the severity and duration of ischemia. Early studies found that an ischemia-induced reliance on glucose oxidation can, paradoxically, be met with inhibition of glycolysis [22]. The production of lactate and feedback inhibition induced by glycolytic products, including PEP, coupled with a decrease in $\mathrm{pH}$, were thought to be the primary means of decreasing glycolysis in the ischemic heart [22]. Thus, ensuring efficient flux through glycolysis has been shown to be a potential point of intervention in attenuating ischemia and reperfusion injury $[24,26]$. In light of our results, it can be seen that an increase in PEP concentration and a drop in ATP may contribute to reversibly inhibiting oxidative phosphorylation. This in turn may initially have a protective effect on limiting electron transport chain activity and preventing free radical production. However, depending upon the severity of ischemia, sustaining high NADH levels may be detrimental. This is because a more reduced environment can increase superoxide production and may ultimately contribute to subsequent reperfusion injury. Future studies will be aimed at defining the molecular mechanisms of PEP-mediated mitochondrial modulation and identifying its contribution to limiting and/or promoting ischemia and reperfusion injury.

\section{Acknowledgments}

This work was supported by the Oklahoma Center for the Advancement of Science and Technology, project \# HR09-139.

\section{References}

[1] W.C. Stanley, F.A. Recchia, G.D. Lopaschuk, Physiol. Rev. 85 (2005) 10931129.

[2] A.L. Shug, E. Shrago, Biochem. Biophys. Res. Commun. 53 (1973) 659-665.

[3] H.S. Sul, E. Shrago, A.L. Shug, Arch. Biochem. Biophys. 172 (1976) 230-237.

[4] J. Nolte, D. Brdiczka, D. Pette, Biochim. Biophys. Acta 284 (1972) 497-507.

[5] B.H. Robinson, FEBS Lett. 14 (1971) 309-312.

[6] J. Yang, S.C. Kalhan, R.W. Hanson, J. Biol. Chem. 284 (2009) 27025-27029.

[7] P. Chudapongse, Biochim. Biophys. Acta 423 (1976) 196-202.

[8] P. Chudapongse, N. Haugaard, Biochim. Biophys. Acta 307 (1973) 599-606.

[9] K.M. Broekemeier, M.E. Dempsey, D.R. Pfeiffer, J. Biol. Chem. 264 (1989) 78267830.

[10] G.D. McCoy, K.A. Doeg, Biochem. Biophys. Res. Commun. 46 (1972) 1411 1417.

[11] G.D. McCoy, K.A. Doeg, J. Biol. Chem. 250 (1975) 3510-3514.

[12] P.W.D. Scisłowski, Mol. Cell. Biochem. 18 (1977) 93-99.

[13] R.W. Estabrook, Methods Enzymol. 10 (1967) 41-47.

[14] R.W. Estabrook, Anal. Biochem. 4 (1962) 231-245.

[15] M.W. Ward, A.C. Rego, B.G. Frenguelli, D.G. Nicholls, J. Neurosci. 20 (2000) 7208-7219.

[16] M.D. Moser, S. Matsuzaki, K.M. Humphries, Arch. Biochem. Biophys. 488 (2009) 69-75.

[17] A.V. Ushakova, V.G. Grivennikova, T. Ohnishi, A.D. Vinogradov, Biochim. Biophys. Acta 1409 (1999) 143-153.

[18] M.P. Murphy, Biochem. J. 417 (2009) 1-13.

[19] L. Wojtczak, A.B. Wojtczak, L. Ernster, Biochim. Biophys. Acta 191 (1969) 1021.

[20] F.L. Muller, Y. Liu, M.A. Abdul-Ghani, M.S. Lustgarten, A. Bhattacharya, Y.C. Jang, H. Van Remmen, Biochem. J. 409 (2008) 491-499.

[21] S. Matsuzaki, L.I. Szweda, K.M. Humphries, Arch. Biochem. Biophys. 484 (2009) 87-93.

[22] M.J. Rovetto, W.F. Lamberton, J.R. Neely, Circ. Res. 37 (1975) 742-751.

[23] D. Sun, N. Nguyen, T.R. DeGrado, M. Schwaiger, F.C. Brosius 3rd, Circulation 89 (1994) 793-798.

[24] G.D. Lopaschuk, W.C. Stanley, Circulation 95 (1997) 313-315.

[25] L.H. Young, Y. Renfu, R. Russell, X. Hu, M. Caplan, J. Ren, G.I. Shulman, A.J. Sinusas, Circulation 95 (1997) 415-422.

[26] N.A. Trueblood, R. Ramasamy, L.F. Wang, S. Schaefer, Am. J. Physiol. Heart Circ. Physiol. 279 (2000) H764-771. 